Pacific

Journal of

Mathematics

APPROXIMATION ENTROPIES IN CROSSED PRODUCTS WITH AN APPLICATION TO FREE SHIFTS

Nathanial P. Brown and Marie Choda 


\title{
APPROXIMATION ENTROPIES IN CROSSED PRODUCTS WITH AN APPLICATION TO FREE SHIFTS
}

\author{
Nathanial P. Brown and Marie Choda
}

\begin{abstract}
It is shown that for two dynamical approximation entropies (one $C^{*}$ and one $W^{*}$ ) the implementing inner automorphism in a crossed product $A \rtimes_{\alpha} \mathbb{Z}$ has the same entropy value as the automorphism $\alpha$.

Using the techniques in the proof, an example of a highly ergodic non-asymptotically abelian automorphism with topological entropy zero is also given. More specifically, it is shown that the free shifts on the Cuntz algebra $\mathcal{O}_{\infty}$ and the reduced free group $C^{*}$-algebra $C_{r}^{*}\left(\mathbb{F}_{\infty}\right)$ have topological entropy zero.
\end{abstract}

\section{Introduction.}

In this paper we show that for two definitions of dynamical entropy (both based on Voiculescu's approximation approach; [Vo2]) getting calculations for general automorphisms is equivalent to getting calculations for inner automorphisms. More precisely, we show that if $\alpha \in \operatorname{Aut}(A), \eta \in S(A)$ is an $\alpha$-invariant state and $\eta \circ E$ denotes the $\operatorname{Ad} u$-invariant state on $A \rtimes_{\alpha} \mathbb{Z}$ induced by $\eta\left(u \in A \rtimes_{\alpha} \mathbb{Z}\right.$ is the implementing unitary) then the entropies of $\alpha$ and $\operatorname{Ad} u$ agree with respect to $\eta$ and $\eta \circ E$, respectively, for the entropy quantities defined in [Ch3] and [Vo2, Section 3]. (See [St2, Problem 4.2].)

One may regard $A \rtimes_{\alpha} \mathbb{Z}$ as the closure of the "fibers" $A_{k}=\left\{a u^{k}: a \in A\right\}$. Then each $A_{k}$ is globally invariant under $\mathrm{Ad} u$ and, moreover, the action of $\operatorname{Ad} u$ on $A_{k}$ is precisely that of $\alpha$. Thus it seems natural to expect the same entropy value for $\alpha$ and $\operatorname{Ad} u$, which we show by constructing explicit completely positive maps on $A \rtimes_{\alpha} \mathbb{Z}$ using the techniques of [SS] as in [Br].

The maps constructed on $A \rtimes_{\alpha} \mathbb{Z}$ can also be used to estimate entropy for some outer automorphisms of $A \rtimes_{\alpha} \mathbb{Z}$. Since many operator algebras can be realized as crossed products we get a large class of examples where these techniques are relevant. Indeed, similar ideas were used in $[\mathbf{C h 2}, \mathbf{C h} 3]$ to obtain various entropy values for Cuntz's canonical endomorphism of the Cuntz algebra $\mathcal{O}_{n}, 2 \leq n<\infty[\mathbf{C u}]$, and Longo's canonical endomorphism of type III factors. In this paper we exploit the isomorphism $\mathcal{O}_{\infty} \otimes \mathcal{K} \cong \mathcal{F} \rtimes_{\Phi} \mathbb{Z}$, where $\mathcal{K}$ denotes the algebra of compact operators and $\mathcal{F}$ is an AF algebra, to obtain the following result. 
Theorem. The free shift on the Cuntz algebra $\mathcal{O}_{\infty}$ has topological entropy zero in the sense of $[\mathbf{V o 2}, \mathbf{B r}]$.

The free shift is the automorphism of $\mathcal{O}_{\infty}=C^{*}\left(\left\{S_{i}: i \in \mathbb{Z}\right\}\right)$ such that $S_{i} \mapsto S_{i+1}$. This is a highly ergodic non-asymptotically abelian automorphism.

There is a natural embedding $C_{r}^{*}\left(\mathbb{F}_{\infty}\right) \hookrightarrow \mathcal{O}_{\infty}$ of the reduced group $C^{*}$ algebra of the free group in infinitely many generators and hence the above theorem also holds for the free shift on $C_{r}^{*}\left(\mathbb{F}_{\infty}\right)$ since topological entropy decreases in subalgebras (cf. [Br, Prop. 2.1]). In fact, we will obtain the same results for automorphisms arising from any bijective function $\mathbb{Z} \rightarrow \mathbb{Z}$. (See also [St1, St3], [Dy] for related results.)

In Section 2 we observe several consequences of the construction of Sinclair and Smith [SS]. The reader is encouraged to first go through $[\mathbf{S S}]$ as we will be rather sketchy. In Section 3 we prove that the entropy of $\alpha$ and $\operatorname{Ad} u$ agree for the entropies defined in [Ch3] and [Vo2, Section 3]. In Section 4 the topological entropy (in the sense of $[\mathbf{B r}]$ ) of the free shift is shown to be zero.

\section{Maps on Crossed Products.}

We first observe that the techniques of [SS] allow one to construct maps on $A \rtimes_{\alpha} G$ out of maps on $A$ in such a way that the map constructed on $A \rtimes_{\alpha} G$ inherits many nice properties that the map on $A$ may have (e.g., normality, positivity, invariance with respect to an $\alpha$-invariant state, approximation properties). For future reference it will be convenient to separate each of these observations into individual propositions. However, all of the results in this section are easy consequences of $[\mathbf{S S}]$ and we refer the reader to that paper for all of the details and notation which appears below.

In this section $A$ will denote a $C^{*}$-algebra which is faithfully nondegenerately represented in $B(H)$, where $H$ is a separable Hilbert space. We assume that an action $\alpha: G \rightarrow \operatorname{Aut}(A)$ is given with $G$ a countable discrete amenable group. As in [SS], we further assume (without loss of generality) that $\alpha$ is spatially implemented; i.e., that there exists a unitary representation $G \rightarrow B(H), g \mapsto U_{g}$ such that $\alpha_{g}(a)=U_{g} a U_{g}^{*}$ for all $a \in A$ and $g \in G$. We will regard $A \rtimes_{\alpha} G$, the reduced (or full, since $G$ is amenable) crossed product, as faithfully represented (via the regular representation) in $B\left(l^{2}(G) \otimes H\right)$ and let $\pi: A \hookrightarrow A \rtimes_{\alpha} G$ denote the natural inclusion. Since $\alpha$ is spatially implemented, the map $\pi$ makes perfectly good sense on all of $B(H)$. An easy calculation shows that $\pi: B(H) \rightarrow B\left(l^{2}(G) \otimes H\right)$ is both ultraweak-ultraweak and ultrastrong-ultrastrong continuous. Recall that there is a natural unitary representation $g \mapsto \lambda_{g}$ of $G$ into $B\left(l^{2}(G) \otimes H\right)$ such that $\lambda_{g} \pi(x) \lambda_{g}^{*}=\pi\left(\alpha_{g}(x)\right)$ for all $x \in A$ and such that the span of $\left\{\pi(x) \lambda_{g}: g \in G, x \in A\right\}$ is norm dense in $A \rtimes_{\alpha} G$. 
If $F \subset G$ is a finite set, we will let $p_{F}$ denote the orthogonal projection onto the span of $\left\{\xi_{g}: g \in F\right\}$ (where $\left\{\xi_{g}\right\}_{g \in G}$ is the natural orthonormal basis of $\left.l^{2}(G)\right)$ and $P_{F}: B\left(l^{2}(G) \otimes H\right) \rightarrow\left(p_{F} \otimes I\right) B\left(l^{2}(G) \otimes H\right)\left(p_{F} \otimes I\right)$ be the compression map. If $f \in l^{\infty}(G)$ has finite support then we let $T_{f}$ : $B\left(l^{2}(G) \otimes H\right) \rightarrow B\left(l^{2}(G) \otimes H\right)$ be the map constructed in [SS, Lem. 3.3].

Definition 2.1. If $\Lambda: A \rightarrow B(H)$ is a linear map, $f \in l^{\infty}(G)$ has finite support, and $F \subset G$ is a finite set we define $\Phi_{\Lambda, f, F}: A \rtimes_{\alpha} G \rightarrow B\left(l^{2}(G) \otimes H\right)$ by

$$
\Phi_{\Lambda, f, F}=T_{f} \circ\left(i d_{F} \otimes \Lambda\right) \circ P_{F},
$$

where $i d_{F}: p_{F}\left(B\left(l^{2}(G)\right)\right) p_{F} \rightarrow p_{F}\left(B\left(l^{2}(G)\right)\right) p_{F}$ is the identity map.

It follows from [SS, Lem. 2.1] that $P_{F}\left(A \rtimes_{\alpha} G\right) \subset p_{F}\left(B\left(l^{2}(G)\right)\right) p_{F} \otimes A$ and hence $\Phi_{\Lambda, f, F}$ is well defined. Since $P_{F}$ is weakly continuous, this also shows that when $A$ is a von Neumann algebra, the weak closure of $A \rtimes_{\alpha} G$ (i.e., the $W^{*}$-crossed product) also gets mapped into $p_{F}\left(B\left(l^{2}(G)\right)\right) p_{F} \otimes A$ and hence $\Phi_{\Lambda, f, F}$ is well defined for $W^{*}$-algebras and $W^{*}$-crossed products as well.

In the following proposition, $I$ will denote the identity operator on both $l^{2}(G)$ and $H$ and hence $I \otimes I$ denotes the unit of $B\left(l^{2}(G) \otimes H\right)$. For each finite set $F \subset G$ we also let $\left\{e_{p, q}\right\}_{p, q \in F}$ denote the canonical matrix units of $p_{F} B\left(l^{2}(G)\right) p_{F}$.

Proposition 2.2. The following assertions hold.

1) $T_{f}$ is a completely positive map $($ cf. $[\mathbf{P a}])$ with $T_{f}(I \otimes I)=\|f\|_{2}^{2} I \otimes I$. Hence $\left\|T_{f}\right\|_{c b}=\left\|T_{f}\right\|=\|f\|_{2}^{2}$. Also, if $F$ contains the support of $f$ then $T_{f}\left(p_{F} \otimes I\right)=T_{f}(I \otimes I)$.

2) $T_{f}\left(e_{p, q} \otimes a\right)=f(p) \overline{f(q)} \pi\left(\alpha_{p}(a)\right) \lambda_{p q^{-1}}$ for $e_{p, q} \otimes a \in p_{F} B\left(l^{2}(G)\right) p_{F} \otimes A$.

3) If $x \in p_{F} B\left(l^{2}(G)\right) p_{F} \otimes A$ and $\left\{x_{i}\right\} \subset p_{F} B\left(l^{2}(G)\right) p_{F} \otimes A$ is a net converging to $x$ in the ultraweak (resp. ultrastrong) topology then $T_{f}\left(x_{i}\right) \rightarrow$ $T_{f}(x)$ in the ultraweak (resp. ultrastrong) topology.

4) If $\Lambda$ is completely bounded (cf. $[\mathbf{P a}])$ then $\Phi_{\Lambda, f, F}$ is also completely bounded with $\left\|\Phi_{\Lambda, f, F}\right\|_{c b} \leq\|f\|_{2}^{2}\|\Lambda\|_{c b}$. If $\Lambda$ is completely positive then $\Phi_{\Lambda, f, F}$ is completely positive.

5) If $A$ is a von Neumann algebra, $\Lambda(A) \subset A$ and $\Lambda$ is ultraweakly (resp. ultrastrongly) continuous then $\Phi_{\Lambda, f, F}$ is ultraweakly (resp. ultrastrongly) continuous as a map $\left(A \rtimes_{\alpha} G\right)^{\prime \prime} \rightarrow B\left(l^{2}(G) \otimes H\right)$.

6) If $A$ is unital, $\Lambda$ is unital, $F \supset \operatorname{supp}(f)$ and $\|f\|_{2}^{2}=1$ then $\Phi_{\Lambda, f, F}$ is unital.

Proof. The first assertion is essentially [SS, Lem. 3.3] and it's proof. (The last statement follows easily from the definition of $T_{f}$.)

The second assertion follows from Lemmas 2.2 and 3.1 in [SS], together with the definition of $T_{f}$. 
The third assertion follows from the second since we noted that $\pi$ is continuous in both the ultraweak and ultrastrong topologies.

The fourth is immediate from the first since $P_{F}$ is a completely positive contraction and $\left\|i d_{F} \otimes \Lambda\right\|_{c b} \leq\|\Lambda\|_{c b}$.

The fifth follows from the third since $\Phi_{\Lambda, f, F}=T_{f} \circ\left(i d_{F} \otimes \Lambda\right) \circ P_{F}$.

The final assertion follows from the first since

$$
\Phi_{\Lambda, f, F}(I \otimes I)=T_{f}\left(i d_{F} \otimes \Lambda\left(p_{F} \otimes I\right)\right)=T_{f}\left(p_{F} \otimes I\right)=T_{f}(I \otimes I)=I \otimes I .
$$

Proposition 2.3. If $\Lambda(A) \subset A$ then

$$
\Phi_{\Lambda, f, F}\left(\pi(a) \lambda_{g}\right)=\sum_{t \in F \cap(g F)} f(t) \overline{f\left(g^{-1} t\right)} \pi\left(\alpha_{t}\left(\Lambda\left(\alpha_{t^{-1}}(a)\right)\right)\right) \lambda_{g}
$$

for all $a \in A$ and $g \in G$. In particular, if $\Lambda(A) \subset A$ then $\Phi_{\Lambda, f, F}\left(A \rtimes_{\alpha} G\right) \subset$ $A \rtimes_{\alpha} G$.

Proof. See Lemma 3.2 in [SS].

Let 1 denote the unit of $G$. Then $P_{\{1\}}\left(A \rtimes_{\alpha} G\right)=p_{\{1\}} \otimes A$. Identifying $p_{\{1\}} \otimes A$ with $I \otimes A \cong A$ we let $E: A \rtimes_{\alpha} G \rightarrow A$ denote the resulting faithful normal projection such that $E\left(\pi(a) \lambda_{g}\right)=0$ for all $g \neq 1$ and $E(\pi(a))=a$ for all $a \in A$ (cf. [Pe, Lem. 7.11.3]). When $A$ is a von Neumann algebra then we regard $E$ as a map $\left(A \rtimes_{\alpha} G\right)^{\prime \prime} \rightarrow A$.

Proposition 2.4. If $\|f\|_{2}^{2}=1, F \supset \operatorname{supp}(f), \eta \in S(A)$ is an $\alpha$-invariant state (i.e., $\eta \circ \alpha_{g}=\eta$ for all $g \in G$ ) and $\Lambda: A \rightarrow A$ is a completely positive map such that $\eta \circ \Lambda=\eta$ then $\eta \circ E \circ \Phi_{\Lambda, f, F}=\eta \circ E\left(\right.$ as states on $\left.A \rtimes_{\alpha} G\right)$.

If $A$ is a von Neumann algebra, $\eta$ is normal and $\Lambda$ is ultraweakly continuous then $\eta \circ E \circ \Phi_{\Lambda, f, F}=\eta \circ E$ (as states on $\left.\left(A \rtimes_{\alpha} G\right)^{\prime \prime}\right)$.

Proof. Evidently Proposition 2.3 implies $\eta \circ E \circ \Phi_{\Lambda, f, F}\left(\pi(a) \lambda_{g}\right)=$ $\eta \circ E\left(\pi(a) \lambda_{g}\right)$ for all $a \in A, g \in G$. Thus the proposition follows from parts 4 and 5 of Proposition 2.2.

The next proposition is immediate from the definitions and part 1 of Proposition 2.2.

Proposition 2.5. Assume $\Lambda=\psi \circ \varphi$ where $\varphi: A \rightarrow B, \psi: B \rightarrow B(H)$ are linear maps. Letting $\Phi=\left(i d_{F} \otimes \varphi\right) \circ P_{F}$ and $\Psi=T_{f} \circ\left(i d_{F} \otimes \psi\right)$ we have:

1) The diagram

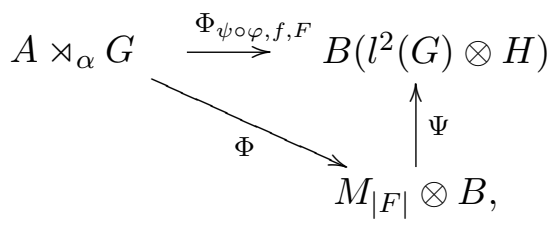


is commutative, where $M_{|F|}=p_{F} B\left(l^{2}(G)\right) p_{F}$ is isomorphic to the matrix algebra of dimension $|F|^{2}=$ cardinality $(F)^{2}$.

2) $\Phi($ resp. $\Psi)$ is completely positive whenever $\varphi($ resp. $\psi)$ is completely positive.

Let $\left\{e_{p, q}\right\}_{p, q \in F}$ be the canonical matrix units of $M_{|F|}=p_{F} B\left(l^{2}(G)\right) p_{F}$. When $\psi(B) \subset A$ we can give an explicit formula for the map $\Psi$. (There is always an explicit formula for $\Phi$.)

Proposition 2.6. With the assumptions and notation of Proposition 2.5 we have:

1. $\Phi\left(\pi(a) \lambda_{g}\right)=\sum_{t \in F \cap(g F)} e_{t, g^{-1} t} \otimes \varphi\left(\alpha_{t^{-1}}(a)\right)$,

$1^{\prime}$. If $\varphi$ is unital then $\Phi$ is unital,

2. $\Psi\left(e_{p, q} \otimes b\right)=f(p) \overline{f(q)} \pi\left(\alpha_{p}(\psi(b))\right) \lambda_{p q^{-1}}$,

$2^{\prime}$. If $F \supset \operatorname{supp}(f),\|f\|_{2}=1$ and $\psi$ is unital then $\Psi$ is unital.

Proof. The first assertion follows from [SS, Lem. 2.1] while 2 follows from the definition of $T_{f}$, [SS, Lem. 3.1] and [SS, Lem. 2.2]. $1^{\prime}$ (resp. $2^{\prime}$ ) is an easy calculation using 1 (resp. 2).

We will need the following proposition to compute [Ch3] entropy.

Proposition 2.7. If $\varphi$ and $\psi$ in Proposition 2.5 are unital and completely positive, $\|f\|_{2}^{2}=1, F \supset \operatorname{supp}(f)$ and $\eta \in S(A)$ is an $\alpha$-invariant state then

$$
\eta \circ E \circ \Psi\left(\sum_{q \in F} e_{q, q} \otimes b\right)=\eta \circ \psi(b),
$$

for all $b \in B$ (i.e., under the natural identifications of $B$ and $1 \otimes B \subset$ $M_{|F|} \otimes B$, the states $\eta \circ \psi$ and $\eta \circ E \circ \Psi$ agree).

Proof. This is an easy calculation using the previous proposition.

Finally we observe that $\Phi_{\Lambda, f, F}$ has good approximation properties whenever $\Lambda$ does. If $K \subset G$ is a finite set and $f \in l^{\infty}(G)$ has finite support $F$ then we let

$$
F_{K, f}=F \cup\left(\bigcup_{g \in K} g^{-1} F\right) .
$$

Proposition 2.8. For each finite set $K \subset G$ and $\delta>0$ there exists $f \in$ $l^{\infty}(G)$ of finite support with $\|f\|_{2}=1$ and the following property: Let $\omega \subset A$ be a finite set with $\|x\| \leq 1$ for all $x \in \omega$. If $\|\Lambda(y)-y\| \leq \delta / 2$ for all $y \in$ $\cup_{g \in F_{K, f}} \alpha_{g^{-1}}(\omega)$ then $\left\|\Phi_{\Lambda, f, F_{K, f}}\left(\pi(x) \lambda_{k}\right)-\pi(x) \lambda_{k}\right\| \leq \delta$ for all $x \in \omega, k \in K$. Proof. If $\Lambda(A) \subset A$ then this is essentially contained in the proof of [SS, Thm. 3.4]. However, a slightly different series of estimates handles the general case (see the proof of $[\mathbf{B r}$, Lem. 3.4]). 
Assume that $\eta \in S(A)$ is an $\alpha$-invariant state and consider the seminorm $\|x\|_{\eta}=\eta\left(x^{*} x\right)^{1 / 2}$ for all $x \in A$. Then $\eta \circ E$ is a state on $A \rtimes_{\alpha} G$ which is $\operatorname{Ad} \lambda_{g}$-invariant for all $g \in G$. An easy calculation shows $\left\|\pi(a) \lambda_{g}\right\|_{\eta \circ E}=\|a\|_{\eta}$ for all $a \in A$ and $g \in G$. Similarly one shows $\left\|\alpha_{t}(a)\right\|_{\eta}=\|a\|_{\eta}$ for all $a \in A$ and $t \in G$. From this it follows that if $\Lambda(A) \subset A$ then

$$
\left\|\Lambda\left(\alpha_{t^{-1}}(a)\right)-\alpha_{t^{-1}}(a)\right\|_{\eta}=\left\|\pi\left(\alpha_{t} \circ \Lambda \circ \alpha_{t^{-1}}(a)\right) \lambda_{g}-\pi(a) \lambda_{g}\right\|_{\eta \circ E},
$$

for all $a \in A$ and $g, t \in G$. However, with this observation the estimates in the proof of [SS, Thm. 3.4] go through essentially without change. Hence we get the following analogue of the previous proposition.

Proposition 2.9. For each finite set $K \subset G$ and $\delta>0$ there exists $f \in$ $l^{\infty}(G)$ of finite support with $\|f\|_{2}=1$ and the following property: Let $\eta \in S(A)$ be an $\alpha$-invariant state and $\omega \subset A$ be a finite set with $\|x\|_{\eta} \leq 1$ for all $x \in \omega$. If $\|\Lambda(y)-y\|_{\eta} \leq \delta / 2$ for all $y \in \cup_{g \in F_{K, f}} \alpha_{g^{-1}}(\omega)$ then $\left\|\Phi_{\Lambda, f, F_{K, f}}\left(\pi(x) \lambda_{k}\right)-\pi(x) \lambda_{k}\right\|_{\eta \circ E} \leq \delta$ for all $x \in \omega, k \in K$.

\section{Entropy and Inner Automorphisms.}

We will now establish the analogue of [Br, Thm. 3.5] for the dynamical entropies defined in [Ch3] and [Vo2, Section 3]. In this section, $\alpha$ will always denote an action of a countable discrete abelian group $G$ on a given operator algebra. Crossed products (both $C^{*}$ and $W^{*}$ ) will be regarded as subalgebras of $B\left(l^{2}(G) \otimes H\right.$ ) (as in the previous section), $\pi: A \rightarrow A \rtimes_{\alpha} G$ is the natural inclusion and $E: A \rtimes_{\alpha} G \rightarrow A$ is the natural faithful normal conditional expectation.

We begin with the analogues of [Br, Lem. 3.4]. The next lemma is used to compute $[\mathbf{C h} \mathbf{3}]$ entropy in crossed products. We refer the reader to $[\mathbf{C h} \mathbf{3}]$ and [Vo2, Section 3] for the definitions and notation which appears below.

Lemma 3.1. Let $A$ be a unital nuclear $C^{*}$-algebra (cf. [Wa]) and $\eta \in S(A)$ be an $\alpha$-invariant state (i.e., $\eta \circ \alpha_{g}=\eta$ for all $g \in G$ ). For each finite set $K \subset G$ and $\delta>0$ there exists a finite set $F=F(K, \delta) \subset G$ such that if $\omega \subset A$ is a finite set with $\|x\| \leq 1$ for all $x \in \omega$ then

$$
\operatorname{scp}_{\eta \circ E}\left(\omega_{K}, \delta\right) \leq s c p_{\eta}\left(\bigcup_{g \in F} \alpha_{-g}(\omega), \delta / 2\right)+\log (|F|),
$$

where $\omega_{K}=\left\{\pi(x) \lambda_{k}: x \in \omega, k \in K\right\}$ and $|F|=\operatorname{cardinality}(F)$.

Proof. Apply Proposition 2.8 with $K, \delta$ to get a function $f \in l^{2}(G)$ with finite support, $\|f\|_{2}=1$ and the property stated in that proposition. We will show that $F=F_{K, f}$ is the desired finite set.

To prove the inequality we let $\varepsilon>0$ be arbitrary and choose unital completely positive maps $\varphi: A \rightarrow B, \psi: B \rightarrow A$ such that $B$ is finite dimensional, $\|\psi \circ \varphi(y)-y\| \leq \delta / 2$ for all $y \in \cup_{g \in F} \alpha_{-g}(\omega)$ and $S(\eta \circ \psi) \leq$ 
$\operatorname{scp}_{\eta}\left(\bigcup_{g \in F} \alpha_{-g}(\omega), \delta / 2\right)+\varepsilon$. Letting $\Lambda=\psi \circ \varphi$ we can factor $\Phi_{\Lambda, f, F}$ (as $\Psi \circ \Phi)$ through $M_{|F|} \otimes B$ by Proposition 2.5. Note also that $\Phi$ and $\Psi$ are unital completely positive maps by Propositions 2.5 and 2.6. Proposition 2.8 says that $\left\|\Phi_{\Lambda, f, F}(x)-x\right\| \leq \delta$ for all $x \in \omega_{K}$ and thus (by definition) $s c p_{\eta \circ E}\left(\omega_{K}, \delta\right) \leq S(\eta \circ E \circ \Psi)$. By [OP, Prop. 1.9] we have

$$
S(\eta \circ E \circ \Psi) \leq S\left(\left.\eta \circ E \circ \Psi\right|_{1 \otimes B}\right)+\log (|F|) .
$$

Finally, from Proposition 2.7, we have $S\left(\left.\eta \circ E \circ \Psi\right|_{1 \otimes B}\right)=S(\eta \circ \psi) \leq$ $\operatorname{scp}_{\eta}\left(\bigcup_{g \in F} \alpha_{-g}(\omega), \delta / 2\right)+\varepsilon$, by our choice of $\psi$, which proves the lemma since $\varepsilon$ was arbitrary.

The next lemma allows one to compute the entropy of [Vo2, Section 3] in crossed products. The proof is similar to the previous one and will be omitted (see also [Br, Lem. 3.4]). Due to the definitions involved, one uses Proposition 2.9 instead of Proposition 2.8. The replacement of the inequality

$$
S(\eta \circ E \circ \Psi) \leq S\left(\left.\eta \circ E \circ \Psi\right|_{1 \otimes B}\right)+\log (|F|)
$$

is the remark that if $\operatorname{rank}(C)$ denotes the dimension of a maximal abelian subalgebra of $C$ then $\operatorname{rank}\left(M_{n}(\mathbb{C}) \otimes B\right) \leq n \cdot \operatorname{rank}(B)$. We also note that one must appeal to Proposition 2.4 to ensure that the maps used in the previous proof (i.e., $\Phi_{\Lambda, f, F}$ ) remain $\eta \circ E$-invariant. (Though not explicitly stated in [Vo2, Section 3], it follows from the assumptions that the state is faithful and the approximating maps in $C P A(M, \eta)$ are $\eta$-invariant that the maps in $C P A(M, \eta)$ are ultraweakly continuous and hence the hypotheses of Proposition 2.4 are indeed satisfied.)

Lemma 3.2. Let $M$ be a hyperfinite von Neumann algebra with $\alpha$-invariant faithful normal state $\eta$. For each finite set $K \subset G$ and $\delta>0$ there exists a finite set $F=F(K, \delta) \subset G$ such that if $\omega \subset M$ is a finite set with $\|x\|_{\eta} \leq 1$ for all $x \in \omega$ then

$$
r c p_{\eta \circ E}\left(\omega_{K}, \delta\right) \leq|F| r c p_{\eta}\left(\bigcup_{g \in F} \alpha_{-g}(\omega), \delta / 2\right),
$$

where $\omega_{K}=\left\{\pi(x) \lambda_{k}: x \in \omega, k \in K\right\}$ and $|F|=\operatorname{cardinality}(F)$.

As in the previous section, we let $\lambda_{g} \in A \rtimes_{\alpha} G$ be the unitary implementing $\alpha_{g} \in \operatorname{Aut}(A)$. We also remind the reader that $G$ is now assumed to be a discrete abelian group.

Theorem 3.3. If $A$ is a unital nuclear $C^{*}$-algebra with $\alpha$-invariant state $\eta$, then for all $g \in G$ we have $h t_{\eta}\left(\alpha_{g}\right)=h t_{\eta \circ E}\left(\operatorname{Ad} \lambda_{g}\right)$, where $h t_{\eta}(\cdot)$ is defined in $[\mathbf{C h} 3]$.

Proof. We only sketch the argument as it is similar to the proof of $[\mathrm{Br}$, Thm. 3.5]. The inequality $h t_{\eta}\left(\alpha_{g}\right) \leq h t_{\eta \circ E}\left(\operatorname{Ad} \lambda_{g}\right)$ for all $g \in G$ follows from [Ch3, Prop. 2.2]. 
Let $\delta>0, \omega \subset A$ a finite set with $\|x\| \leq 1$ for all $x \in \omega$, and a finite set $K \subset G$ be given. Choose a finite set $F=F(K, \delta)$ according to Lemma 3.1 and define $\Omega=\cup_{g \in F} \alpha_{-g}(\omega)$. Since $G$ is abelian, from Lemma 3.1 one may deduce the inequality

$\operatorname{scp}_{\eta \circ E}\left(\omega_{K} \cup \ldots \cup \operatorname{Ad} \lambda_{g}^{n-1}\left(\omega_{K}\right), \delta\right) \leq s c p_{\eta}\left(\Omega \cup \ldots \cup \alpha_{g}^{n-1}(\Omega), \delta / 2\right)+\log (|F|)$,

as in the proof of $[\mathbf{B r}$, Thm. 3.5]. Since this inequality holds for all $n \in \mathbb{N}$, the desired inequality follows from [Ch3, Prop. 2.3].

The proof of the following theorem is similar where one uses Lemma 3.2 instead of Lemma 3.1. The analogues of [Ch3, Prop. 2.2] and [Ch3, Prop. 2.3] are [Vo2, Prop. 3.5] and [Vo2, Prop. 3.4], respectively. Of course, $\operatorname{Ad} \lambda_{g}$ should now be regarded as an automorphism of the $W^{*}$-crossed product.

Theorem 3.4. Let $M$ be a hyperfinite von Neumann algebra and $\eta$ be an $\alpha$-invariant faithful normal state. For all $g \in G$, we have $h c p a_{\eta}\left(\alpha_{g}\right)=$ $h c p a_{\eta \circ E}\left(\operatorname{Ad} \lambda_{g}\right)$, where $h c p a_{\eta}(\cdot)$ is defined in [Vo2, Section 3].

In particular, this theorem generalizes the results of [Vo2, Appendix].

\section{Entropy for Automorphisms of $\mathcal{O}_{\infty}$.}

In this section we will show that $h t(\alpha)=0$ (cf. [Br]) for the free shifts on $\mathcal{O}_{\infty}$ and $C_{r}^{*}\left(\mathbb{F}_{\infty}\right)$. This will follow from a more general result concerning automorphisms of $\mathcal{O}_{\infty}$ induced by bijective mappings $\alpha: \mathbb{Z} \rightarrow \mathbb{Z}$. These results have also been obtained by K. Dykema (cf. [Dy, Thm. 1 and Example 7]) using directly the free product construction as opposed to the crossed product construction used here. As mentioned in the introduction, we use the isomorphism $\mathcal{O}_{\infty} \otimes \mathcal{K} \cong \mathcal{F} \rtimes_{\Phi} \mathbb{Z}$ and the techniques of the previous two sections to achieve our calculations.

Recall that the Cuntz algebra $\mathcal{O}_{\infty}$ is defined as the universal $C^{*}$-algebra generated by isometries $\left\{S_{i}\right\}_{i \in \mathbb{Z}}$ which satisfy the relation

$$
\sum_{i=-r}^{r} S_{i} S_{i}^{*} \leq 1
$$

for all $r \in \mathbb{N}$. If $\alpha: \mathbb{Z} \rightarrow \mathbb{Z}$ is any bijective function, then from the universality of $\mathcal{O}_{\infty}$ we get a well defined automorphism $\mathcal{O}_{\infty} \rightarrow \mathcal{O}_{\infty}$ defined by $S_{i} \mapsto S_{\alpha(i)}$. We will also use $\alpha$ to denote the automorphism of $\mathcal{O}_{\infty}$ induced by $\alpha: \mathbb{Z} \rightarrow \mathbb{Z}$. If $\alpha$ is the mapping $i \mapsto i+1$ then $\alpha$ is called the free shift.

We begin with a technical lemma which should have appeared in $[\mathbf{B r}]$ and will be necessary for our calculations. If $A \subset B(H)$ we will let $\iota_{A}$ denote the inclusion $A \hookrightarrow B(H)$. See [Br, Def. 1.1] for the notation which appears below. 
Lemma 4.1. Let $C, D \subset B(H)$ be exact $C^{*}$-algebras (cf. [Wa]) and $\pi$ : $C \rightarrow D$ be $a{ }^{*}$-monomorphism. For each finite set $\omega \subset C$ and $\delta>0$, $\operatorname{rcp}\left(\iota_{C}, \omega, \delta\right)=\operatorname{rcp}\left(\iota_{D}, \pi(\omega), \delta\right)$.

Proof. From the proofs of [Br, Prop. 1.3 and 2.14] we have $\operatorname{rcp}\left(\iota_{C}, \omega, \delta\right)=$ $r c p(\pi, \omega, \delta)$ and $r c p\left(\iota_{D}, \pi(\omega), \delta\right)=r c p\left(\iota_{\pi(C)}, \pi(\omega), \delta\right)$. Hence it is sufficient to show $\operatorname{rcp}(\pi, \omega, \delta)=\operatorname{rcp}\left(\iota_{\pi(C)}, \pi(\omega), \delta\right)$.

We only show $\operatorname{rcp}(\pi, \omega, \delta) \geq \operatorname{rcp}\left(\iota_{\pi(C)}, \pi(\omega), \delta\right)$ as the other inequality is similar. So choose $(\varphi, \psi, B) \in C P A(\pi, C)$ such that $\|\psi \circ \varphi(x)-\pi(x)\| \leq$ $\delta$ for all $x \in \omega$ and $\operatorname{rank}(B)=\operatorname{rcp}(\pi, \omega, \delta)$. Then $\left(\varphi \circ \pi^{-1}, \psi, B\right) \in$ $C P A\left(\iota_{\pi(C)}, \pi(C)\right)$ and $\left\|\psi \circ \varphi \circ \pi^{-1}(\pi(x))-\pi(x)\right\| \leq \delta$ for all $x \in \omega$. But this implies $r c p(\pi, \omega, \delta) \geq \operatorname{rcp}\left(\iota_{\pi(C)}, \pi(\omega), \delta\right)$ as desired.

Remark 4.2. In particular, this lemma improves [Br, Lem. 2.4] and hence the proofs of Propositions 2.5, 2.6 and 2.8 in [Br] are slightly more technical than they need to be.

Given $n \in \mathbb{N}$ and a subset $I \subset \mathbb{Z}$ we let $W(n, I)=\left\{\mu=\left(\mu_{1}, \ldots, \mu_{n}\right)\right.$ : $\mu_{j} \in I$ for $\left.1 \leq j \leq n\right\}$ and $W(0, I)=\{\emptyset\}$. If $\mu \in W(n, I)$ we define the operator $S_{\mu} \in \mathcal{O}_{\infty}$ by $S_{\mu}=S_{\mu_{1}} \cdots S_{\mu_{n}}$ and $S_{\mu}=1$ if $n=0$. For $m \leq n$ we let $[m, n]$ be the integer interval and $\mathcal{F}([m, n], I)$ be the $C^{*}$-subalgebra of $\mathcal{O}_{\infty}$ generated by

$$
\bigcup_{j=m}^{n}\left\{S_{\mu} S_{\nu}^{*}: \mu, \nu \in W(j, I)\right\} .
$$

It is known that if $I$ is a finite subset of $\mathbb{Z}$ then $\mathcal{F}(n, I)=\mathcal{F}([n, n], I)$ is isomorphic to the matrix algebra $M_{|I|^{n}}(\mathbb{C})$, where $|I|$ denotes the cardinality of $I$, and is isomorphic to the compact operators on an infinite dimensional separable Hilbert space when $I$ is infinite $(\mathrm{cf}$. $[\mathbf{C u}]$ ). If $I \subset \mathbb{Z}$ is a finite set, we define for each $j \in \mathbb{N}$ the projection

$$
P_{j}=\sum_{\mu \in W(j, I)} S_{\mu} S_{\mu}^{*}
$$

Note that $P_{j} \geq P_{j+1}$.

Lemma 4.3. If $I \subset \mathbb{Z}$ is a finite set then

$$
\mathcal{F}([0, n], I) \cong \mathbb{C} \oplus M_{|I|}(\mathbb{C}) \oplus \ldots \oplus M_{|I|^{n}}(\mathbb{C})
$$

with a complete set of pairwise orthogonal minimal projections given by

$$
\left\{S_{\mu}\left(1-P_{1}\right) S_{\mu}^{*}, S_{\nu} S_{\nu}^{*}: \mu \in \cup_{j \in[0, n-1]} W(j, I) \text { and } \nu \in W(n, I)\right\} .
$$

In particular, $\operatorname{rank}(\mathcal{F}([0, n], I))=1+|I|+\cdots+|I|^{n}$. 
Proof. For each $j$, the set $\left\{S_{\mu} S_{\nu}^{*}: \mu, \nu \in W(j, I)\right\}$ is a complete set of matrix units for $\mathcal{F}(j, I)$ and the unit of $\mathcal{F}(j, I)$ is $P_{j}$. For each $0 \leq j \leq n-1$ we define

$$
E_{j}(\mu, \nu)=S_{\mu}\left(1-P_{1}\right) S_{\nu}^{*}
$$

where $\mu, \nu \in W(j, I)$. For $j=n$ we let

$$
E_{n}(\mu, \nu)=S_{\mu} S_{\nu}^{*}
$$

where $\mu, \nu \in W(n, I)$. For each $0 \leq j \leq n$ let $A_{j}=C^{*}\left(\left\{E_{j}(\mu, \nu): \mu, \nu \in\right.\right.$ $W(j, I)\})$. (Note that $A_{n}=\mathcal{F}(n, I)$.) Evidently we have $A_{j} \subset \mathcal{F}([0, n], I)$ for $0 \leq j \leq n$ and hence $C^{*}\left(\left\{A_{j}: 0 \leq j \leq n\right\}\right) \subset \mathcal{F}([0, n], I)$. Note also that for $\mu, \nu \in W(n-1, I), S_{\mu} S_{\nu}^{*}=E_{n-1}(\mu, \nu)+S_{\mu} P_{1} S_{\nu}^{*}$ and hence $S_{\mu} S_{\nu}^{*} \in A_{n-1}+A_{n}$. Similarly one argues that $S_{\mu} S_{\nu}^{*} \in A_{j}+\cdots+A_{n}$ for $\mu, \nu \in W(j, I)$ and hence $C^{*}\left(\left\{A_{j}: 0 \leq j \leq n\right\}\right)=\mathcal{F}([0, n], I)$.

For $0 \leq j \leq n-1$ a simple calculation shows $E_{j}(\mu, \nu) E_{j}\left(\mu^{\prime}, \nu^{\prime}\right)=$ $\delta_{\nu, \mu^{\prime}} E_{j}\left(\mu, \nu^{\prime}\right)$ and $\sum_{\mu} E_{j}(\mu, \mu)=P_{j}-P_{j+1}$. One also verifies that for $0 \leq$ $j \leq n-1$ and $\mu, \nu \in W(j, I), S_{\mu}^{*} P_{j+1}=P_{1} S_{\mu}^{*}$. Hence $P_{j+1} S_{\mu} S_{\nu}^{*}=S_{\mu} S_{\nu}^{*} P_{j+1}$ and

$$
\left(P_{j}-P_{j+1}\right) S_{\mu} S_{\nu}^{*}\left(P_{j}-P_{j+1}\right)=S_{\mu}\left(1-P_{1}\right) S_{\nu}^{*}=E_{j}(\mu, \nu),
$$

for $0 \leq j \leq n-1$ and $\mu, \nu \in W(j, I)$. Thus $\left\{E_{j}(\mu, \nu): \mu, \nu \in W(j, I)\right\}$ is a complete set of matrix units for $A_{j}=\left(P_{j}-P_{j+1}\right) \mathcal{F}(j, I)\left(P_{j}-P_{j+1}\right) \cong$ $M_{|I|^{j}}(\mathbb{C})$. This also shows that the $A_{j}$ are pairwise orthogonal and hence

$$
\mathcal{F}([0, n], I)=C^{*}\left(\left\{A_{j}: 0 \leq j \leq n\right\}\right)=A_{0} \oplus \ldots \oplus A_{n} .
$$

However, this clearly implies the lemma.

Since $\mathcal{F}([0, n],[-n, n]) \subset \mathcal{F}([0, n+1],[-n-1, n+1]) \subset \mathcal{O}_{\infty}$, the closure $\mathcal{F}_{\infty}$ of $\cup_{n} \mathcal{F}([0, n],[-n, n])$ is an AF subalgebra containing the unit of $\mathcal{O}_{\infty}$.

For each $i \in \mathbb{Z}$ let $B_{i}=\mathcal{F}_{\infty}$ and define ${ }^{*}$-monomorphisms $\beta_{i, i-1}: B_{i} \rightarrow$ $B_{i-1}$ by $x \mapsto S_{0} x S_{0}^{*}$. Let $B$ denote the inductive limit of the sequence

$$
\cdots B_{1} \stackrel{S_{0} \cdot S_{0}^{*}}{\hookrightarrow} B_{0} \stackrel{S_{0} \cdot S_{0}^{*}}{\hookrightarrow} B_{-1} \stackrel{S_{0} \cdot S_{0}^{*}}{\hookrightarrow} \cdots
$$

and $\rho_{i}: B_{i} \hookrightarrow B$ be the induced embeddings. As in $[\mathbf{C u}]$, there is an automorphism $\Phi$ of $B$ which shifts the above sequence one space to the left and satisfies the relation

$$
\Phi^{j} \circ \rho_{i}=\rho_{i+j},
$$

for all $i, j \in \mathbb{Z}$ (under the natural identifications $B_{i} \cong \mathcal{F}_{\infty} \cong B_{i+j}$ ). Another important relation that follows immediately from the construction is

$$
\rho_{i}(x)=\rho_{i-j}\left(S_{0}^{j} x S_{0}^{* j}\right),
$$

for all $x \in B_{i} \cong \mathcal{F}_{\infty} \cong B_{i-j}, i \in \mathbb{Z}$ and $j \in \mathbb{N}$. 
Let $u$ be the implementing unitary in the multiplier algebra $M\left(B \rtimes_{\Phi} \mathbb{Z}\right)$. One readily verifies that the elements $\tilde{S}_{i}=\rho_{0}\left(S_{i} S_{0}^{*}\right) u \in B \rtimes_{\Phi} \mathbb{Z}$ are partial isometries with support projection $\rho_{0}(1)$ and satisfying

$$
\sum_{i=-r}^{r} \tilde{S}_{i} \tilde{S}_{i}^{*} \leq \rho_{0}(1)
$$

for all $r \in \mathbb{N}$. Hence there is an induced ${ }^{*}$-monomorphism $\pi: \mathcal{O}_{\infty} \rightarrow B \rtimes_{\Phi} \mathbb{Z}$ such that $\pi\left(S_{i}\right)=\tilde{S}_{i}$ for all $i \in \mathbb{Z}$. In $[\mathbf{C u}]$, it is shown that $\pi\left(\mathcal{O}_{\infty}\right)=$ $\rho_{0}(1)\left(B \rtimes_{\Phi} \mathbb{Z}\right) \rho_{0}(1)$. However, for our calculations, it will only be necessary to observe that for every $x \in \mathcal{F}_{\infty}$ we have $\pi(x)=\rho_{0}(x)$.

We will use this map $\pi$ (and Lemma 4.1) to estimate the completely positive $\delta$-rank of certain finite sets in $\mathcal{O}_{\infty}$. To compute $\delta$-ranks in crossed products (i.e., $B \rtimes_{\Phi} \mathbb{Z}$ ) we recall [Br, Lem. 3.4] (in a slightly different, but equivalent, form than the original).

Lemma 4.4 ([Br, Lem. 3.4]). Let $A \subset B(H)$ be an exact $C^{*}$-algebra ( $c f$. $[\mathbf{W a}])$ and $\alpha: G \rightarrow \operatorname{Aut}(A)$ be an action of a discrete abelian group. For each finite set $K \subset G$ and $\delta>0$ there exists a finite set $F=F(K, \delta) \subset G$ such that if $\omega \subset A$ is a finite set with $\|x\| \leq 1$ for all $x \in \omega$ then

$$
r c p\left(i d_{A \rtimes_{\alpha} G}, \omega_{K}, \delta\right) \leq|F| r c p\left(i d_{A}, \bigcup_{g \in F} \alpha_{-g}(\omega), \delta / 2\right),
$$

where $\omega_{K}=\left\{\pi(x) \lambda_{k}: x \in \omega, k \in K\right\}$ and $|F|=\operatorname{cardinality}(F)$.

Considering the case $G=\mathbb{Z}$, what this lemma roughly says is that to approximate polynomials in $A \rtimes_{\alpha} \mathbb{Z}$ it suffices to approximate a finite number of iterates of the coefficients.

For convenience, we assume both $\mathcal{O}_{\infty}$ and $B \rtimes_{\Phi} \mathbb{Z}$ to be faithfully represented on the same Hilbert space $H$ and use $\iota_{\mathcal{O}_{\infty}}$ and $\iota_{B \rtimes_{\Phi} \mathbb{Z}}$ to denote the inclusions. It will also be convenient to define

$$
\omega_{k, l, I}=\bigcup_{i=0}^{k}\left(\bigcup_{j=0}^{l}\left\{S_{0}^{* i} S_{\mu} S_{\nu}^{*}, S_{\mu} S_{\nu}^{*} S_{0}^{i}: \mu, \nu \in W(j, I)\right\}\right) .
$$

Lemma 4.5. For each $k, l \in \mathbb{N}$ with $k \leq l$ and $\delta>0$ there is a constant $C=C(k, l, \delta)$ such that $\operatorname{rcp}\left(\iota_{\mathcal{O}_{\infty}}, \omega_{k, l, I}, \delta\right) \leq C|I|^{C}$ for all finite subsets $I \subset \mathbb{Z}$ containing 0 .

Proof. Note that $\pi\left(S_{0}^{i}\right)=\rho_{0}\left(S_{0}^{i} S_{0}^{* i}\right) u^{i}$. Hence for all $i \leq k$ and $\mu, \nu \in W(j, I)$ we have

$$
\pi\left(S_{\mu} S_{\nu}^{*} S_{0}^{i}\right)=\rho_{0}\left(S_{\mu} S_{\nu}^{*} S_{0}^{i} S_{0}^{* i}\right) u^{i}=\rho_{-k}\left(S_{0}^{k} S_{\mu} S_{\nu}^{*} S_{0}^{i} S_{0}^{* i} S_{0}^{* k}\right) u^{i}
$$

and

$$
\pi\left(S_{0}^{* i} S_{\mu} S_{\nu}^{*}\right)=u^{* i} \rho_{0}\left(S_{0}^{i} S_{0}^{* i} S_{\mu} S_{\nu}^{*}\right)
$$


Since $u^{*} \rho_{0}(x)=\rho_{-1}(x) u^{*}$ we have

$$
\pi\left(S_{0}^{* i} S_{\mu} S_{\nu}^{*}\right)=\rho_{-i}\left(S_{0}^{i} S_{0}^{* i} S_{\mu} S_{\nu}^{*}\right) u^{* i}=\rho_{-k}\left(S_{0}^{k-i} S_{0}^{i} S_{0}^{* i} S_{\mu} S_{\nu}^{*} S_{0}^{*(k-i)}\right) u^{* i} .
$$

Thus we see that the "coefficients" of $\pi\left(\omega_{k, l, I}\right)$ (i.e., $\rho_{-k}\left(S_{0}^{k} S_{\mu} S_{\nu}^{*} S_{0}^{i} S_{0}^{*(k+i)}\right)$ and $\left.\rho_{-k}\left(S_{0}^{k} S_{0}^{* i} S_{\mu} S_{\nu}^{*} S_{0}^{*(k-i)}\right)\right)$ all come from the finite dimensional algebra $\rho_{-k}(\mathcal{F}([0, l+k], I))$ by reducing the terms $S_{\nu}^{*} S_{0}^{i}$ and $S_{0}^{* i} S_{\mu}$.

By virtue of Lemmas 4.1 and 4.4, to estimate $\operatorname{rcp}\left(\iota_{\mathcal{O}_{\infty}}, \omega_{k, l, I}, \delta\right)$ it suffices to understand a finite number of the iterates (under $\Phi$ ) of the coefficients of $\pi\left(\omega_{k, l, I}\right)$. But since there is always a conditional expectation onto finite dimensional subalgebras, we only need to understand a finite number of iterates of the finite dimensional subalgebra $\rho_{-k}(\mathcal{F}([0, l+k], I)$ ) (since this contains the coefficients of $\left.\pi\left(\omega_{k, l, I}\right)\right)$.

So let $m \in \mathbb{N}$ be arbitrary and consider

$$
\Phi^{m}\left(\rho_{-k}(\mathcal{F}([0, l+k], I))\right) \cup \ldots \cup \Phi^{-m}\left(\rho_{-k}(\mathcal{F}([0, l+k], I))\right) .
$$

By the relations $\Phi^{j} \circ \rho_{-k}=\rho_{-k+j}$, and $\rho_{t}(x)=\rho_{t-r}\left(S_{0}^{r} x S_{0}^{* r}\right)(r \geq 0)$ we have

$$
\begin{aligned}
& \Phi^{m}\left(\rho_{-k}(\mathcal{F}([0, l+k], I))\right) \cup \ldots \cup \Phi^{-m}\left(\rho_{-k}(\mathcal{F}([0, l+k], I))\right) \\
& =\rho_{-k+m}(\mathcal{F}([0, l+k], I)) \cup \ldots \cup \rho_{-k-m}(\mathcal{F}([0, l+k], I)) \\
& =\rho_{-k-m}\left(S_{0}^{2 m} \mathcal{F}([0, l+k], I) S_{0}^{*(2 m)} \cup \ldots \cup \mathcal{F}([0, l+k], I)\right) \\
& \subset \rho_{-k-m}(\mathcal{F}([0, l+k+2 m], I)) .
\end{aligned}
$$

Hence, by Lemmas 4.1, 4.4 and our observations above, there exists $m=$ $m(k, \delta) \in \mathbb{N}$ such that

$$
\begin{aligned}
\operatorname{rcp}\left(\iota_{\mathcal{O}_{\infty}}, \omega_{k, l, I}, \delta\right) & \leq(2 m+1) \operatorname{rank}\left(\rho_{-k-m}(\mathcal{F}([0, l+k+2 m], I))\right) \\
& =(2 m+1) \operatorname{rank}(\mathcal{F}([0, l+k+2 m], I)) \\
& =(2 m+1)\left(1+|I|+\cdots+|I|^{(l+k+2 m)}\right) \\
& \leq C|I|^{C}
\end{aligned}
$$

where $C=l+k+2 m+1$.

Since $\mathcal{O}_{\infty}$ is nuclear, the following theorem holds for the entropy defined in $[\mathbf{V o 2}$, Section 4] although we will be using the definition in $[\mathbf{B r}]$ (cf. $[\mathbf{B r}$, Prop. 1.4]).

Theorem 4.6. If $\alpha \in \operatorname{Aut}\left(\mathcal{O}_{\infty}\right)$ is induced by a bijective function $\alpha: \mathbb{Z} \rightarrow \mathbb{Z}$ then $h t(\alpha)=0$. 
Proof. If $I \subset \mathbb{Z}$ contains $0, \mu \in W(j, I)$ and $\nu \in W\left(j^{\prime}, I\right)$, where $j \leq j^{\prime}$, then $S_{\mu} S_{\nu}^{*}=S_{0}^{* i} S_{\gamma} S_{\nu}^{*}$ with $\gamma \in W\left(j^{\prime}, I\right)$ and $i=j^{\prime}-j$. From this observation and a similar remark when $j>j^{\prime}$, one deduces that the span of the finite sets $\omega_{k, l, I}$ with $k \leq l$ and $0 \in I$ are norm dense in $\mathcal{O}_{\infty}$. Hence it suffices, by $\left[\right.$ Br, Prop. 2.6], to show that $h t\left(\iota_{\mathcal{O}_{\infty}}, \alpha, \omega_{k, l, I}, \delta\right)=0$ for all such sets.

If $\mu, \nu \in W(j, I)$ then an easy calculation shows $\alpha\left(S_{\mu} S_{\nu}^{*} S_{0}^{i}\right)=S_{\gamma} S_{\lambda}^{*} S_{0}^{i}$ for some $\gamma, \lambda \in W(j,\{0\} \cup \alpha(I))$ if $j \geq i$ or $\gamma, \lambda \in W(i,\{0\} \cup \alpha(I))$ if $j<i$. In any case, one deduces that $\alpha\left(\omega_{k, l, I}\right) \subset \omega_{k, l,\{0\} \cup \alpha(I)}$ whenever $k \leq l$. Similarly one shows

$$
\omega_{k, l, I} \cup \ldots \cup \alpha^{n-1}\left(\omega_{k, l, I}\right) \subset \omega_{k, l, I \cup \ldots \cup \alpha^{n-1}(I)},
$$

for all $n \in \mathbb{N}$, whenever $k \leq l$ and $0 \in I \subset \mathbb{Z}$.

Hence

$$
r c p\left(\iota_{\mathcal{O}_{\infty}}, \omega_{k, l, I} \cup \ldots \cup \alpha^{n-1}\left(\omega_{k, l, I}\right), \delta\right) \leq r c p\left(\iota_{\mathcal{O}_{\infty}}, \omega_{k, l, I \cup \ldots \cup \alpha^{n-1}(I)}, \delta\right),
$$

for all $n$ and all $\delta>0$. However, the previous lemma shows that

$$
\operatorname{rcp}\left(\iota_{\mathcal{O}_{\infty}}, \omega_{k, l, I \cup \ldots \cup \alpha^{n-1}(I)}, \delta\right) \leq C(n|I|)^{C}
$$

for some constant $C$ depending only on $k, l$ and $\delta$. This implies

$$
h t\left(\iota_{\mathcal{O}_{\infty}}, \alpha, \omega_{k, l, I}, \delta\right) \leq \limsup _{n \rightarrow \infty} n^{-1}\left(\log \left(C(n|I|)^{C}\right)\right)=0 .
$$

Remark 4.7. Note that we have never used the fact that $\alpha: \mathbb{Z} \rightarrow \mathbb{Z}$ is surjective. Thus the previous theorem also holds for any endomorphism of $\mathcal{O}_{\infty}$ which is induced by an injective function $\alpha: \mathbb{Z} \rightarrow \mathbb{Z}$.

The following recovers a special case of [St3, Thm. 2] in the case of CNT entropy.

Corollary 4.8. Let $\alpha \in \operatorname{Aut}\left(\mathcal{O}_{\infty}\right)$ be induced by a bijective function $\alpha$ : $\mathbb{Z} \rightarrow \mathbb{Z}$ and $\varphi$ be an $\alpha$-invariant state. Then $h_{\varphi}(\alpha)=h t_{\varphi}(\alpha)=0$, where $h_{\varphi}(\cdot)$ and $h t_{\varphi}(\cdot)$ are defined in $[\mathbf{C N T}]$ and $[\mathbf{C h 3}]$, respectively.

Proof. Since $\mathcal{O}_{\infty}$ is nuclear we appeal to $[\mathbf{C h} 3$, Thm. 2.6.1] to get the inequalities $h t(\alpha) \geq h t_{\varphi}(\alpha) \geq h_{\varphi}(\alpha)$.

If $\mathbb{F}_{\infty}$ is the free group on generators $\left\{g_{i}\right\}_{i \in \mathbb{Z}}$ then $C_{r}^{*}\left(\mathbb{F}_{\infty}\right)$ is the $C^{*}$ algebra generated by the left regular representation $\lambda: \mathbb{F}_{\infty} \rightarrow B\left(l^{2}\left(\mathbb{F}_{\infty}\right)\right)$. If $\alpha: \mathbb{Z} \rightarrow \mathbb{Z}$ is any bijective function then there is a natural automorphism of $C_{r}^{*}\left(\mathbb{F}_{\infty}\right)$, also denoted by $\alpha$, such that $\lambda\left(g_{i}\right) \mapsto \lambda\left(g_{\alpha(i)}\right)$. The free shift on $C_{r}^{*}\left(\mathbb{F}_{\infty}\right)$ is induced by the mapping $i \mapsto i+1$. 
Corollary 4.9. Let $\alpha \in \operatorname{Aut}\left(C_{r}^{*}\left(\mathbb{F}_{\infty}\right)\right)$ be induced by a bijective function $\alpha: \mathbb{Z} \rightarrow \mathbb{Z}$. Then $h t(\alpha)=0$.

Proof. By monotonicity of $h t(\cdot)$ (i.e., the fact that topological entropy decreases in invariant subalgebras; cf. [Br, Prop. 2.1]) it suffices to provide an embedding of $C_{r}^{*}\left(\mathbb{F}_{\infty}\right)$ into $\mathcal{O}_{\infty}$ such that $\alpha$ lifts to an automorphism of $\mathcal{O}_{\infty}$ of the type considered in Theorem 4.6. That such an embedding exists is known to the experts so we only sketch the proof. (See also [BD].)

Let $\left\{S_{i}\right\}_{i \in \mathbb{Z}}$ generate $\mathcal{O}_{\infty}$ and $\varphi \in S\left(\mathcal{O}_{\infty}\right)$ be the vacuum state (cf. [VDN, Ex. 1.5.8]). The restriction of $\varphi$ to $A_{i}=C^{*}\left(S_{i}\right)$ is denoted by $\varphi_{i}$. Let $B_{i} \subset A_{i}$ be the subalgebra generated by the identity and $b_{i}=\left(S_{i}^{*}+S_{i}\right) / 2$. Then the distribution of $b_{i}$ with respect to $\varphi_{i}$ is the semicircular law, $\gamma_{0,1}$ (cf. [VDN, Def. 2.6.1]). Thus each $B_{i}$ is isomorphic to $C([-1,1])$ and

$$
\varphi_{i}\left(b_{i}^{n}\right)=\frac{2}{\pi} \int_{-1}^{1} t^{n} \sqrt{1-t^{2}} d t,
$$

for all $n \in \mathbb{N}$. The unitary $v \in C([-1,1])$ defined by $v(t)=\exp (2 i(\arcsin t+$ $\left.\sqrt{1-t^{2}}\right)$ ) satisfies

$$
\frac{2}{\pi} \int_{-1}^{1} v^{n}(t) \sqrt{1-t^{2}} d t=0,
$$

for all nonzero $n \in \mathbb{Z}$. Hence each $B_{i}$ contains a unitary $u_{i}$ with $\varphi_{i}\left(u_{i}^{n}\right)=0$ for all nonzero $n \in \mathbb{Z}$. Then $C^{*}\left(\left\{u_{i}: i \in \mathbb{Z}\right\}\right)$ is isomorphic to $C_{r}^{*}\left(\mathbb{F}_{\infty}\right)$ and $\alpha$ lifts to an automorphism of $\mathcal{O}_{\infty}$ under this identification.

Acknowledgements. This work grew out of a visit by the first named author to Osaka Kyoiku University while supported at the University of Tokyo by an NSF Dissertation Enhancement Award. He would like to thank that institution for it's support and hospitality. He would also like to thank Y. Kawahigashi for some helpful comments.

Corollary 4.9 was obtained from discussions with K. Dykema while the second named author participated in the January 1999 seminar "Complex Function Theory and Functional Analysis" organized by J.B. Cooper at the Erwin Schrodinger Institute. She would like to express her hearty thanks to the ESI, the organizers and K. Dykema.

\section{References}

[Av] D. Avitzour, Free products of $C^{*}$-algebras, Trans. Amer. Math. Soc., 271 (1982), 423-435.

[BD] E. Blanchard and K. Dykema, Embeddings of reduced free products of operator algebras, preprint. 
[Br] N.P. Brown, Topological entropy in exact $C^{*}$-algebras, Math. Ann., 314 (1999), 347-367.

[Ch1] M. Choda, Reduced free products of completely positive maps and entropy for free products of automorphisms, Publ. RIMS, Kyoto Univ., 32 (1996), 371-382.

[Ch2] Entropy of Cuntz's canonical endomorphism, Pac. J. Math., 190 (1999), 235-245.

[Ch3] $\quad A C^{*}$-dynamical entropy and applications to canonical endomorphisms, J. Funct. Anal., 172 (2000), to appear.

[CNT] A. Connes, H. Narnhofer and W. Thirring, Dynamical entropy of $C^{*}$-algebras and von Neumann algebras, Comm. Math. Phys., 112 (1987), 691-719.

[CS] A. Connes and E. Størmer, Entropy of $\mathrm{II}_{1}$ von Neumann algebras, Acta Math., 134 (1975), 289-306.

$[\mathrm{Cu}] \quad$ J. Cuntz, Simple $C^{*}$-algebras generated by isometries, Comm. Math. Phys., 57 (1977), 173-185.

[De] V. Deaconu, Entropy estimates for some $C^{*}$-endomorphisms, Proc. Amer. Math. Soc., 127 (1999), 3653-3658.

[Dy] K. Dykema, Topological entropy for some automorphisms of reduced amalgamated free product $C^{*}$-algebras, preprint.

[Hu] T. Hudetz, Topological entropy for appropriately approximated $C^{*}$-algebras, J. Math. Phys., 35 (1994), 4303-4333.

[OP] M. Ohya and D. Petz, Quantum Entropy and its Use, Texts and Monographs in Physics, Springer-Verlag, Berlin, 1993.

[Pa] V. Paulsen, Completely Bounded Maps and Dilations, Pitman Research Notes in Mathematics, Vol. 146, Longman, 1986.

[Pe] G. Pedersen, $C^{*}$-algebras and Their Automorphism Groups, Academic Press, London, 1979.

[SS] A.M. Sinclair and R.R. Smith, The completely bounded approximation property for discrete crossed products, Indiana Univ. Math. J., 46 (1997), 1311-1322.

[St1] E. Størmer, Entropy of some automorphisms of the $\mathrm{II}_{1}$ factor of the free group in infinite number of generators, Invent. Math., 110 (1992), 63-73.

[St2] E. Størmer, Entropy in operator algebras, Asterisque, 232 (1995), 211-230.

[St3] E. Størmer, States and shifts on infinite free products of $C^{*}$-algebras, Fields Inst. Comm., 12 (1997), 281-291.

[Th] K. Thomsen, Topological entropy for endomorphisms of local $C^{*}$-algebras, Comm. Math. Phys., 164 (1994), 181-193.

[Vo1] D. Voiculescu, Symmetries of Some Reduced Free Product $C^{*}$-algebras, Operator Algebras and their connections with Topology and Ergodic Theory, Lecture Notes in Math., Vol. 1132, Springer-Verlag, 1985, 556-588.

[Vo2] - Dynamical approximation entropies and topological entropy in operator algebras, Comm. Math. Phys., 170 (1995), 249-281.

[VDN] D. Voiculescu, K. Dykema and A. Nica, Free Random Variables, CRM Monograph Series, Amer. Math. Soc., 1992. 
[Wa] S. Wassermann, Exact $C^{*}$-algebras and Related Topics, Lecture Notes Series, 19, GARC, Seoul National University, 1994.

Received July 15, 1999 and revised April 6, 2000. The first author is an NSF Postdoctoral Fellow. The second author was partially supported by the Schrodinger Institute.

Department of Mathematics

UNIVERSITY OF CALIFORNIA

BERKELEY, CA 94720

E-mail address: nbrown@math.berkeley.edu

Department of Mathematics

OSAKA KYOIKU UNIVERSITY

KASHIWARA 582-8582

JAPAN

E-mail address: marie@cc.osaka-kyoiku.ac.jp 